\title{
INHALTSVERZEICHNISS.
}

Seite

Einleitung . . . . . . . . . . . . . . . . . . . . 1-6

Vom Schachbrett und den Zügen . . . . . . . . . . . . . 7-8

Gang und Werth der Steine . . . . . . . . . . . . . . 9-14

Regeln des Schachspiels . . . . . . . . . . . . . . . . 15-18

Eröffnung der Partic . . . . . . . . . . . . . . . . . 19-24

Erste Abtheilung. Eröffnungen auf der Damenseite . . . . . 25-54

Erstes Spiel.

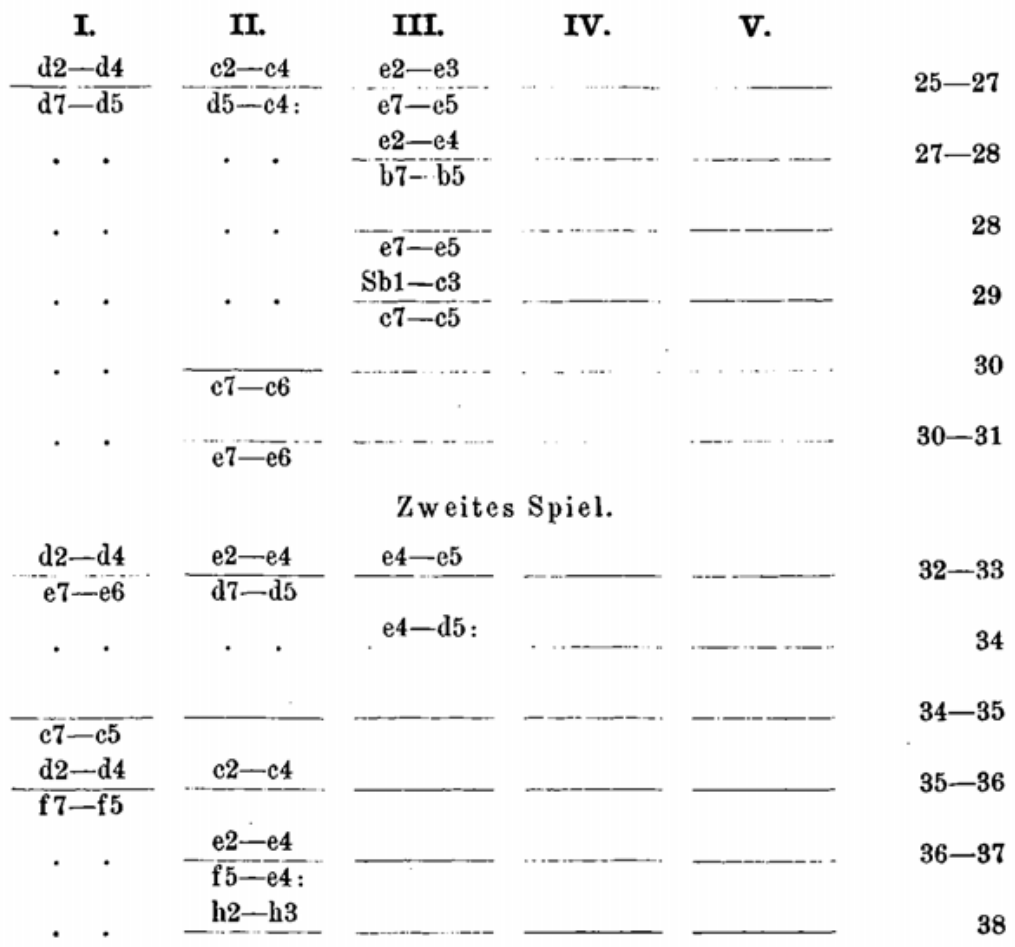


VI

Inhaltsverzeichniss.

I.

II.

$\mathrm{c2}-\mathrm{c} 4$

$\frac{\mathrm{f} 2-\mathrm{f} 4}{\mathrm{~d} 7-\mathrm{d} 5}$

e7-e5

$\frac{\mathrm{a} 2-\mathrm{a} 3}{\mathrm{e} 7-\mathrm{e} 5}$

$\frac{\mathrm{e} 2-\mathrm{e} 4}{\mathrm{e} 7-\mathrm{e} 6}$

. $\quad \mathrm{f} 2-\mathrm{f} 4$

c7-c5

. $\quad \frac{\mathrm{Sg} 1-\mathrm{f3}}{\mathrm{Sb8}-\mathrm{c6}}$

$$
\begin{aligned}
& \mathrm{e} 7-\mathrm{e} 6 \\
& \mathrm{~d} 2-\mathrm{d} 4
\end{aligned}
$$

$\frac{\mathrm{e} 2-\mathrm{e} 4}{\mathrm{~d} 7-\mathrm{d} 5}$

$$
\text { b7-b6 }
$$

$\overline{\mathrm{Sb} 8-\mathrm{c} 6}$
III.

Drittes Spiel.

IV.

v.

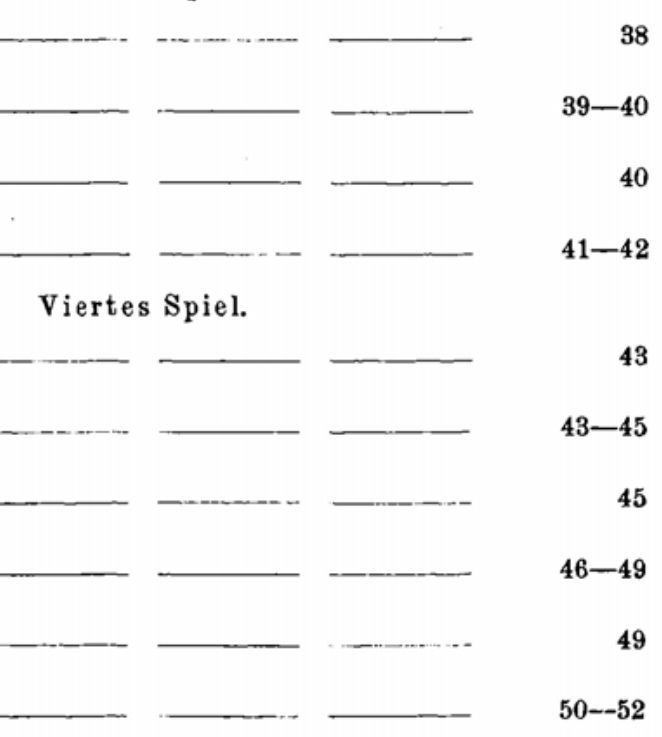

Fünftes Spiel.

Zweite Abtheilung. Gewöhnliche Spiele auf der Königsseite

$55-188$

\section{Erstes Spiel.}

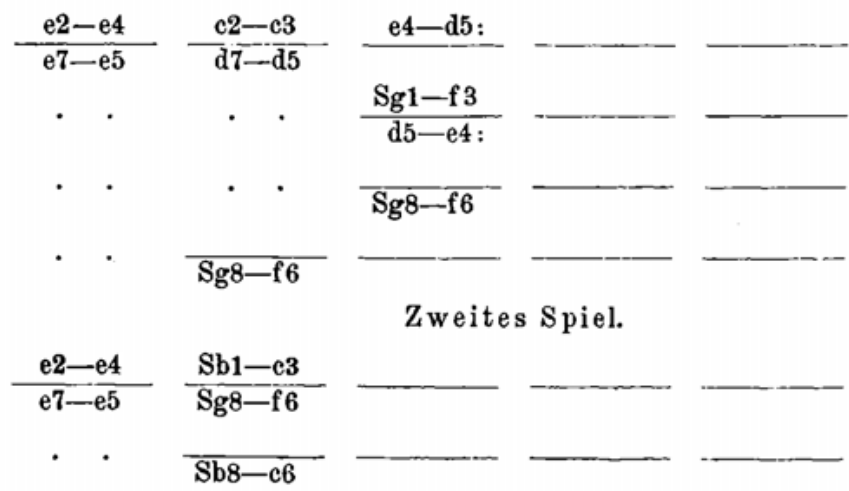

$56-57$

57

58

$58-60$

61 
Inhaltsverzeichniss.

VII

I. II. III. IV. V.

Drittes Spiel.

Seite

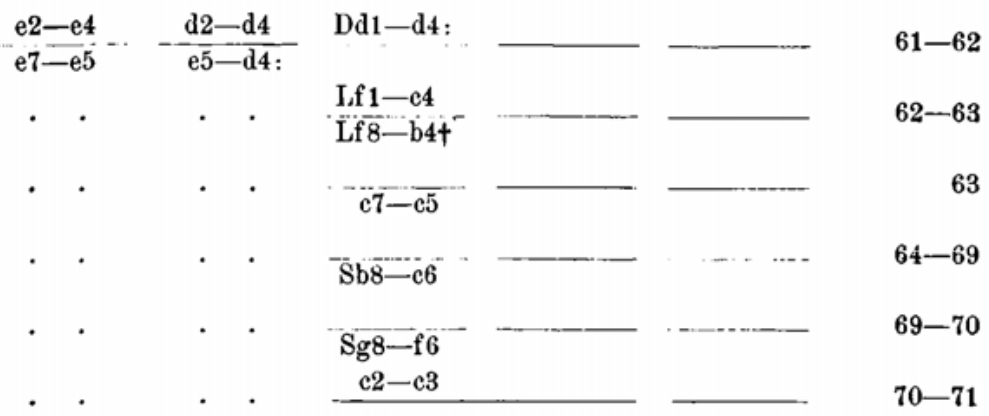

Läuferspiel . . . . . . . . . . . . . . . . . 71-97

Viertes spiel.

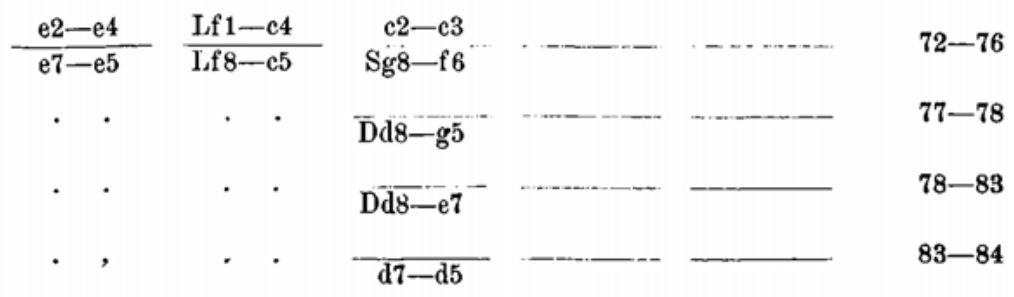

Fünftes Spiel.

$\frac{\mathrm{e} 2-\mathrm{e} 4}{\mathrm{e} 7-\mathrm{e} 5} \quad \frac{\mathrm{Lf} 1-\mathrm{c} 4}{\mathrm{Lf} 8-\mathrm{c} 5} \cdot \frac{\mathrm{Sg} 1-\mathrm{f} 3}{\mathrm{~d} 7-\mathrm{d} 6}$

Sg8-f 6

85

Dd1-h5

85

Dd1-e2

86

$\mathrm{f} 2-\mathrm{f} 4$

$\longrightarrow$

86

Le4-f 7:+

87

$\mathrm{d} 2-\mathrm{d} 4$

87

b2-b4

87 
I. II. III.

IV.

v.

Sechstes Spiel.

$\frac{\mathrm{e} 2-\mathrm{e} 4}{\mathrm{e} 7-\mathrm{e} 5} \quad \frac{\mathrm{Lf} 1-\mathrm{c4}}{\mathrm{Sg} 8-\mathrm{f} 6} \frac{\mathrm{d} 2-\mathrm{d} 3}{\text { Lf } 8-\mathrm{c5}}$

88-90

Sg1-f3

$90-92$

f2-f 4

Siebentes Spiel.

$\frac{\mathrm{e} 2-\mathrm{e} 4}{\mathrm{e} 7-\mathrm{e} 5} \quad \frac{\text { Lf } 1-\mathrm{c} 4}{\mathrm{c7}-\mathrm{c} 6}$

$\mathrm{d} 2-\mathrm{d} 3$

$-\overline{\mathrm{f} 7-\mathrm{f} 5}$

Sg1-f3

Le4-g8:

95- 96

e4-f5:

96-97

Springerspiel

$97-188$

Achtes Spiel.

$\frac{\mathrm{e} 2-\mathrm{e} 4}{\mathrm{e} 7-\mathrm{e} 5} \frac{\mathrm{Sg} 1-\mathrm{f} 3}{\mathrm{f} 7-\mathrm{f} 6}$

Neuntes Spiel.

$\frac{\mathrm{e} 2-\mathrm{e} 4}{\mathrm{e} 7-\mathrm{e} 5} \frac{\mathrm{Sg} 1-\mathrm{f} 3}{\mathrm{f} 7-\mathrm{f} 5}$

$\frac{e 2-e 4}{e 7-e 5}-\frac{S g 1-f 3}{d 7-d 5}$

Zehntes Spiel.

98

98-99

$99-101$

Elftes Spiel.

$\frac{\mathrm{e} 2-\mathrm{e} 4}{\mathrm{e} 7-\mathrm{e} 5} \quad \frac{\mathrm{Sg} 1-\mathrm{f} 3}{\mathrm{~d} 7-\overline{\mathrm{d} 6}}-\frac{\mathrm{d} 2-\mathrm{d} 4}{\mathrm{f} 7-\mathrm{f} 5}$

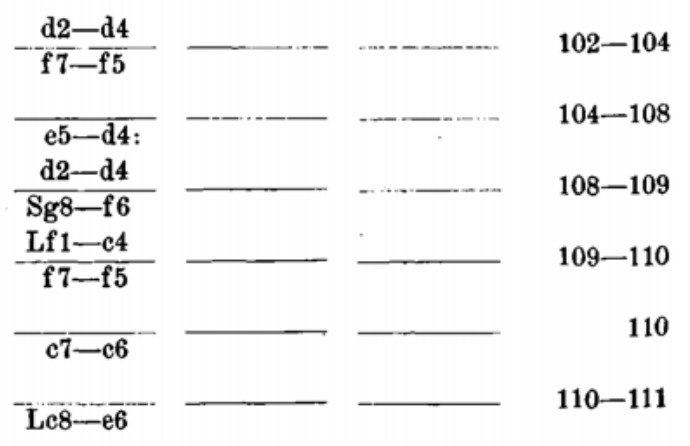


Inhaltsverzeichniss.

IX

I. II, III. IV. V.

¿wölftes Spiel. Seite

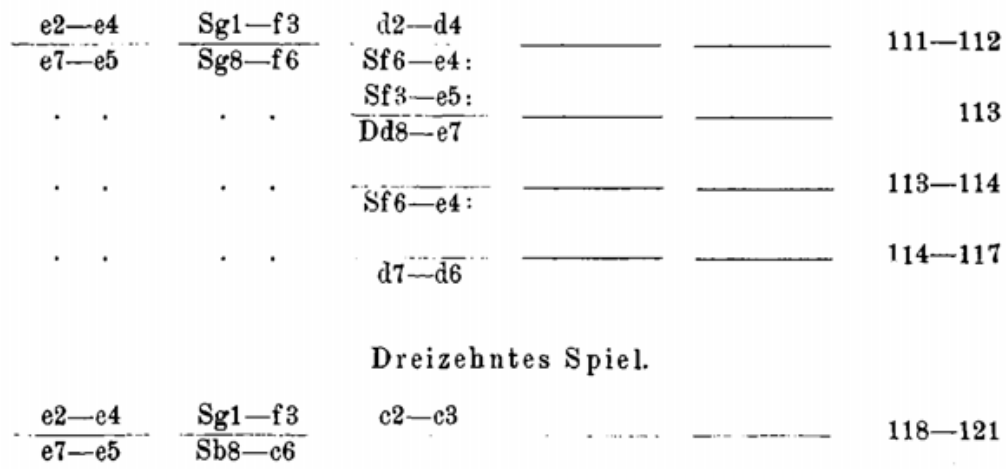

Vierzehntes Spiel.

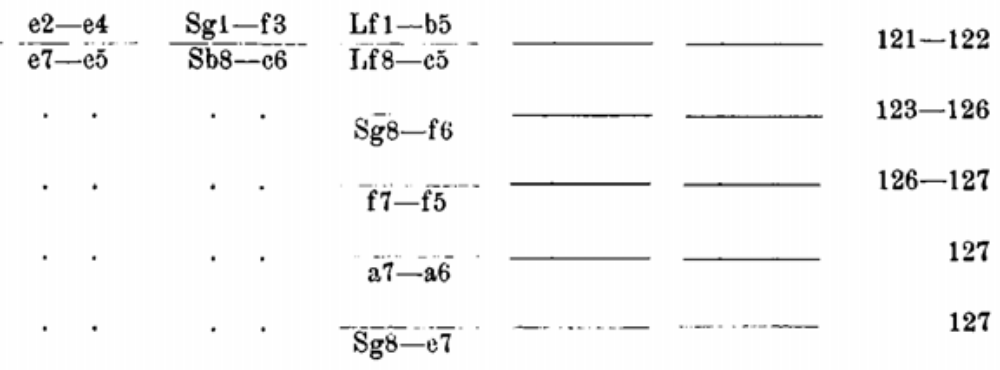

Fünfzehntes Spiel.

$\frac{\mathrm{e} 2-\mathrm{e} 4}{\mathrm{e} 7-\mathrm{e} 5}-\frac{\mathrm{Sg} 1-\mathrm{f} 3}{\mathrm{Sb} 8-\mathrm{c} 6} \quad \frac{\mathrm{Lf} 1-\mathrm{c} 4}{\mathrm{f} 7-\mathrm{f} 5}$

$127-128$

Sechszehntes Spiel.

$\begin{array}{llllll}\mathrm{e} 2-\mathrm{e} 4 & \mathrm{Sg} 1-\mathrm{f} 3 & \mathrm{Lf} 1-\mathrm{c} 4 & \mathrm{Sf} 3-\mathrm{g} 5 & & 128-134 \\ \mathrm{e} 7-\mathrm{e} 5 & \mathrm{Sb8}-\mathrm{c} 6 & \mathrm{Sg} 8-\mathrm{f} 6 & \frac{\mathrm{d} 7-\mathrm{d} 5}{2} & \cdots & \end{array}$

Sf $6-\mathrm{e} \overline{4} \quad 135$

Siebenzehntes Spiel.

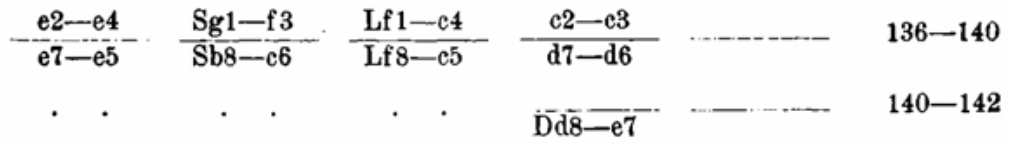


Inhaltsverzeichniss.

I.

II.

$$
\begin{aligned}
& \text { III. IV. }
\end{aligned}
$$

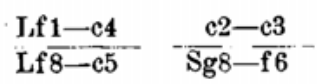

$$
\begin{aligned}
& \text { f } 7 \text { - } 5 \\
& 0-0 \\
& \text { Sg8-f } 6
\end{aligned}
$$

v.

$\frac{e^{2}-e 4}{e 7-e 5} \frac{\mathrm{Sg} 1-\mathrm{f} 3}{\mathrm{Sb8}-\mathrm{c} 6}$

Achtzehntes Spiel.

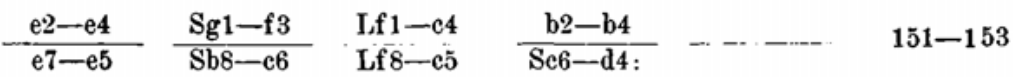

$$
\begin{aligned}
& \text { Le5-b4: } \quad c 2-c 3 \quad 153-155
\end{aligned}
$$

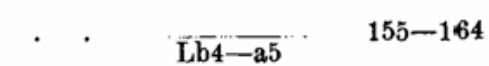

$$
\begin{aligned}
& \text {. } \quad \frac{164-169}{\mathrm{Lb} 4-\mathrm{C5}} \quad 169 \\
& \mathrm{Lc} 5-\mathrm{b} 6{ }^{-}-\cdots-169-172 \\
& \text { d7-d5 } \quad 172-173
\end{aligned}
$$

Neunzehntes Spiel.

$\frac{\mathrm{e} 2-\mathrm{e} 4}{\mathrm{e} 7-\mathrm{e}} \cdots \cdot \frac{\mathrm{Sg} 1-\mathrm{f} 3}{\mathrm{Sb} 8-\mathrm{c} 6} \quad \frac{\mathrm{d} 2-\mathrm{d} 4}{\mathrm{Se} 6-\mathrm{d} 4:}$

$$
\text { Sf } 3-\mathrm{d} 4
$$

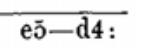

Sf $3-d 4$ :

$174-176$

Lf $1-\mathrm{c} 4$

$\overline{\mathrm{D}} \mathrm{d} 8-\mathrm{f} 6 \mathrm{cos}$

$176-177$

Iff $8-\mathrm{c5}$

178-182

Lf $8-b 4+$

$182-188$

Dritte Abtheilung. Gambit des Königs . . . . . . . . 189-256

Erstes Spiel.

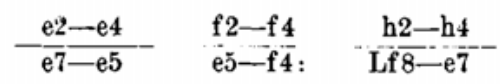

Läufergambit . . . . . . . . . . . . . . . . . . 191-210

Zweites Spiel.

$\frac{\mathrm{e} 2-\mathrm{e} 4}{\mathrm{e} 7-\mathrm{e} 5} \quad \frac{\mathrm{f2}-\mathrm{f} 4}{\mathrm{e} 5-\mathrm{f} 4:} \frac{\mathrm{Lf} 1-\mathrm{e4}}{\mathrm{Dd} 8-\mathrm{h} 4 \dagger}-\frac{\mathrm{Ke} 1-\mathrm{f} 1}{\mathrm{~d} 7-\mathrm{d} 6}$ $191-195$ 
Inhaltsverzeichniss.

I. II. III. IV.

Drittes Spiel. Seite

$\begin{array}{llll}\mathrm{e} 2-\mathrm{e} 4 & \mathrm{f} 2-\mathrm{f} 4 & \text { Iff } 1-\mathrm{ct} & \mathrm{Ke} 1-\mathrm{f} 1 \\ \mathrm{e} 7-\mathrm{e} 5 & \mathrm{e} 5-\mathrm{f} 4: & \text { Dd8-h4t } & -\frac{\mathrm{g} 7-\mathrm{g} 5}{}\end{array}$

$195-203$

Viertes Spicl.

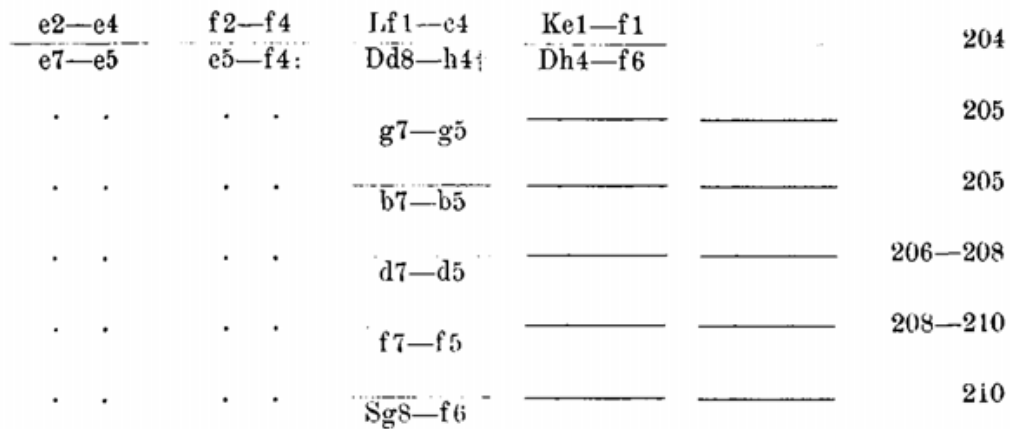

Springergambit . . . . . . . . . . . . . . . . 211-251

Fünftes Spiel.

$\begin{array}{rrrrr}\mathrm{e} 2-\mathrm{e} 4 & \mathrm{f} 2-\mathrm{f} 4 & \mathrm{Sg} 1-\mathrm{f} 3 & & \\ -\mathrm{e} 7-\mathrm{e} 5 & \mathrm{e} 5-\mathrm{f} 4: & \mathrm{Iff}-\mathrm{e} 7 & - & 212-213\end{array}$

Sechstes Spiel.

\begin{tabular}{ccccc}
$\mathrm{e} 2-\mathrm{e} 4$ & $\mathrm{f} 2-\mathrm{f} 4$ \\
\hdashline $\mathrm{e} 7-\mathrm{e} 5$ & $\mathrm{e} 5-\mathrm{f} 4:$ & $\frac{\mathrm{Sg} 1-\mathrm{f} 3}{\mathrm{~g} 7-\mathrm{g} 5}$ & $\frac{\mathrm{h} 2-\mathrm{h} 4}{\mathrm{~g} 5}-\mathrm{g} 4$ & $\mathrm{Sf} 3-\mathrm{g} 5$ \\
$\mathrm{~h} 7-\mathrm{h} 6$ & $\mathbf{2 1 4 - 2 1 5}$
\end{tabular}

$\begin{array}{cr}\mathrm{Sf} 3-\mathrm{e} 5 \\ \mathrm{~h} 7-\mathrm{h} 5\end{array} \quad 215-218$

$-\bar{d} 7-\mathrm{d} 6-218-219$

Lf $8-\mathrm{e} 7 \quad 219-220$

Sg8-f $6 \quad 220-222$

If $8-g 7 \quad 222-224$

Siebentes Spiel.

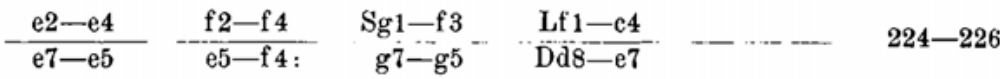

226 
I.

II.
III.

Achtes Spiel.
$\frac{\mathrm{Sg} 1-\mathrm{f} 3}{\mathrm{~g} 7-\mathrm{g} 5}-\frac{\mathrm{Lf} 1-\mathrm{c} 4}{\mathrm{Lf} 8-\mathrm{g} 7}$

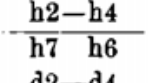

$-\frac{\mathrm{d} 2-\mathrm{d} 4}{\mathrm{~d} 7-\mathrm{d} 6}$

Neuntes Spiel.

$\frac{\mathrm{Sg} 1-\mathrm{f} 3}{\mathrm{~g} 7-\mathrm{g} 5}$

$\frac{\text { Lf } 1-\mathrm{c} 4}{\mathrm{~g} 5-\mathrm{g} 4}$

$\frac{\text { Sf } 3-\mathrm{e} 5}{\text { Dd8-h4+ }}$

$235-240$

Zehntes Spiel.

$$
\begin{aligned}
& \frac{\mathrm{e} 2-\mathrm{e} 4}{\mathrm{e} 7-\mathrm{e} 5} \quad \frac{\mathrm{f} 2-\mathrm{f} 4}{\mathrm{e} 5-\mathrm{f} 4:} \quad \frac{\mathrm{Sg} 1-\mathrm{f} 3}{\mathrm{~g} 7-\mathrm{g} 5} \quad \frac{\mathrm{Lf} 1-\mathrm{c} 4}{\mathrm{~g} 5-\mathrm{g} 4} \quad \begin{array}{l}
\text { Le4-f } 7: \dagger \\
\mathrm{Ke} 8-\mathrm{f} 7:
\end{array} \\
& \text { g- } 0 \\
& \frac{\mathrm{d} 2-\mathrm{d} 4}{\mathrm{~g} 4-\mathrm{f} 3:} \\
& \frac{\mathrm{Sb1}-\mathrm{c3}}{\mathrm{g} 4-\mathrm{f} 3:}
\end{aligned}
$$

Elftes Spiel.

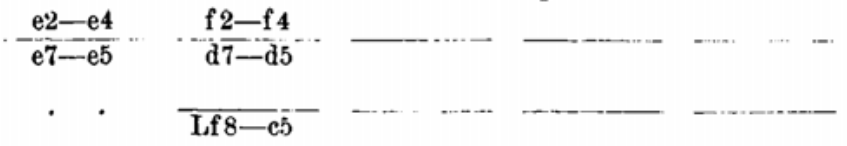

I. Die beiden Läufer gewinnen gegen den einzelnen König .

II. Läufer und Springer gewinnen gegen den König

III. Die Dame gewinnt gegen den Thurm, selbst gegen Thurm und Bauer

259-261

IV. Die Dame gegen beide Läufer . . . . . . . . . . .

V. Die Dame gegen beide Springer . . . . . . . . . . 262-263

VI. Die Dame gegen Läufer und Springer . . . . . . . . 263-264

VII. Thurm gegen Thurm und Läufer . . . . . . . . . . 265-267

VIII. Thurm und Springer gegen Thurm . . . . . . . . . 267-268

IX. Thurm und Bauer gegen Thurm . . . . . . . . . . . 268-269

X. Der Läufer macht das Spiel gegen Thurm, zuweilen gegen

Thurm und Bauer unentschieden . . . . . . . . . 269-272

XI. Thurm gegen Springer. . . . . . . . . . . . . . 272

XII. Der Thurm gegen Officier und zwei Bauern . . . . . . 272-273

XIII. Läufer gegen Springer nebst Bauern . . . . . . . . . 273-274

XIV. Läufer gegen Bauern . . . . . . . . . . . . . . 274-275

XV. König und ein Bauer . . . . . . . . . . . . . . 275-278

XVI. Bauern gegen Bauern . . . . . . . . . . . . . . 278-283 